\title{
Ablação ocular no camarão Macrobrachium rosenbergii (De Man) (Crustacea, Decapoda, Palaemonidae): efeitos sobre a reprodução, pigmentação epidérmica e atividade alimentar
}

\author{
Marcel José Martins dos Santos ${ }^{1}$ \\ Marcelo Antonio Amaro Pinheiro ${ }^{2}$
}

\begin{abstract}
Eyestalk ablation in the prawn Macrobrachium rosenbergii (De Man) (Crustacea, Decapoda, Palaemonidae): effects on reproduction, epidermic pigmentation and feeding activity. This study analyze the consequences of unilateral and bilateral ablation based on ovigerous percentage, consecutive spawns, and secondary effects of the surgical process in the females of Macrobrachium rosenbergii (De Man, 1879). Two experiments were carried out with four and seven months old females in intermolt stage. Each experiment was comprised of control, unilateral and bilateral ablation. Eyestalk ablation was done with a bistoury with a topic hot cauterization followed by application of antibiotic pomades. The animals were maintained at constant temperature $\left(28 \pm 1,05^{\circ} \mathrm{C}\right)$ and photoperiod of $12 \mathrm{~L}: 12 \mathrm{D}$ within fibercement boxes with sandy bottom and biological filter. Females were observed once a day during fourteen weeks, registering gonadal condition, ecdysis and presence of spermatophore (mating) and spawning. Unilateral ablation technique is more efficient due to the anticipation of the first spawn, repeatability between spawns, expressive rate of ovigerous females and survival, that favored its applicability. Bilateral eyestalk ablation produced the mortality of all the females with change in coloration and food activity patterns. These results corroborate other observations on penaeid shrimps, though bilateral ablation on some lobsters was a success. These results showing an interespecific variation and can be used in aquaculture projects.

KEY WORDS. Crustacea, eyestalk ablation, reproduction, pigmentation, feeding activity, prawn
\end{abstract}

A obtenção de pós-larvas do camarão de água doce Macrobrachium rosenbergii (De Man, 1879) é limitada pela baixa disponibilidade de fêmeas ovígeras durante os meses de inverno em regiões de clima subtropical. Quando a temperatura da água dos viveiros é inferior a $18^{\circ} \mathrm{C}$, os camarões apresentam uma diminuição do crescimento somático e da atividade reprodutiva, resultando, muitas vezes, em alta mortalidade. Após a água dos viveiros ganhar calor gradativamente, o cultivo pode ser reiniciado, embora, nesta ocasião, os criadores não possuam um número suficiente de pós-larvas para o povoamento; tal fato se deve à temperatura e fotoperíodo reduzidos, responsáveis pela minimização do processo reprodutivo na maioria dos crustáceos decápodos já estudados (STEELE et al. 1977; PAYEN 1980; CAMPBELL \& Fielder 1986; MEUSY \& PAYEN 1988).

1) Laboratório de Biologia de Crustáceos, Centro de Aqüicultura, Universidade Estadual Paulista.

2) Departamento de Biologia Aplicada, Universidade Estadual Paulista, Núcleo de Estudos em Biologia, Ecologia e Cultivo de Crustáceos. Via de acesso Professor Paulo Donato Castellane, 14884-900 Jaboticabal, São Paulo, Brasil. 
A técnica de ablação do pedúnculo ocular vem sendo utilizada com sucesso na indução da maturação gonadal desses animais (SANTIAGo 1977; PRIMAVERA 1978; LAWRENCE et al. 1980; BROWDy \& SAMOCHA 1985; JuINIO-MENÊZ \& RUINATA 1996; SAGI et al. 1997), obtendo-se uma antecipação do estado ovígero e aumento na freqüência de desovas. TAN-FERMIN (1991) menciona que essa técnica tem sido amplamente empregada no cultivo de camarões peneídeos, particularmente em espécies de difícil reprodução em cativeiro, o que, segundo NEW (1990), já não ocorre comercialmente para Macrobrachium rosenbergii.

A técnica de ablação ocular consiste na extirpação de um ou ambos pedúnculos oculares, podendo ser realizada por dois métodos distintos: 1) incisão no globo ocular do crustáceo com remoção do complexo endócrino por pressão superficial (Primavera 1978); e 2) remoção total ou parcial do pedúnculo ocular com cauterização "a quente", utilizando uma agulha incandescente (PRIMAVERA 1985), ou "a frio", com a aplicação de resina de nitrocelulose (DÉMEUSY 1962) ou nitrogênio líquido (SAGI et al. 1997).

Um dos primeiros trabalhos que avalia a técnica de ablação do pedúnculo ocular em crustáceos decápodos é o de PANOUSE (1943), que verificou uma aceleração expressiva no crescimento ovariano. O pedúnculo ocular dos crustáceos abriga células neuro-secretoras que formam o complexo órgão-X/glândula do seio (COX/GS), responsável pela produção, estocagem e distribuição de hormônios reguladores da muda e gametogênese. Com a ablação do pedúnculo ocular elimina-se a síntese desses neuro-hormônios, promovendo um desequilíbrio hormonal com alterações diretas sobre tais processos (ADIYODI \& ADIYODI 1970).

O órgão-X sintetiza o Hormônio Inibidor Gonadal (HIG) que é armazenado e distribuído pela glândula do seio. Com a retirada do pedúnculo ocular elimina-se a fonte produtora do HIG, permitindo uma maior concentração do Hormônio Estimulador Gonadal (HEG), produzido pelo órgão-Y no cefalotórax, estimulando a maturação gonadal (ADIYODI \& ADIYODI 1970). De acordo com PRIMAVERA (1979), a ablação do pedúnculo ocular deve ser efetuada no estágio de intermuda para que ocorra maturação gonadal, pois, caso contrário, verifica-se a ativação do processo de muda e aumento do período de latência para a próxima maturação. Tal fato é reflexo do antagonismo existente entre os processos de crescimento e reprodução nos crustáceos decápodos, que competem pela mesma fonte energética estocada no hepatopâncreas (KYOMO 1988).

O controle da intensidade de pigmentação e coloração epidérmica também é realizado por neuro-hormônios (cromatoforotropinas), produzidos pelo órgão-x dos crustáceos decápodos (BRown 1935), sendo reflexo quantitativo e qualitativo da dispersão e distribuição dos cromatóforos na epiderme (RAO 1985). A extirpação do pedúnculo ocular causa uma redução desses hormônios na circulação, promovendo mudanças no padrão de coloração característico da espécie (PASTEUR 1958; SHIBLEY 1968).

No pedúnculo ocular também são sintetizadas substâncias reguladoras da alimentação (SEARS et al. 1991), ocorrendo um aumento na ingestão de alimentos na ausência de uma substância peptídica produzida pelo complexo órgão-X/glândula do seio, denominada Fator Inibidor da Alimentação (FIA). 
O presente trabalho tem como objetivo analisar a eficiência da técnica de ablação unilateral e bilateral do pedúnculo ocular em fềmeas de $M$. rosenbergii, com diferente faixa etária, verificando sua influência sobre a reprodução, pigmentação epidérmica e comportamento alimentar.

\section{MATERIAL E MÉTODOS}

Foram montados dois experimentos com camarões provenientes de viveirosberçário, sendo utilizadas no primeiro 15 fêmeas com quatro meses (comprimento total de $58,16 \pm 4,69 \mathrm{~mm}$ e peso de $15,62 \pm 3,69 \mathrm{~g}$ ) e, no segundo, 15 fêmeas com sete meses de idade (comprimento total de $144,00 \pm 0,98 \mathrm{~mm}$ e peso de $31,09 \pm 4,29 \mathrm{~g}$ ).

As fêmeas selecionadas encontravam-se em estádio de intermuda, verificado por inspeção da margem do exopodito do urópodo sob estereomicroscópio, de acordo com DRACH \& TCHERNIGOVTZEFF (1967). Foram estabelecidos três tratamentos: 1) controle; 2) ablação unilateral; e 3) ablação bilateral. Em cada um deles os camarões foram colocados na proporção de um macho BC ("Blue Claw") para cinco fêmeas (1:5), em tanques de fibrocimento de 750 litros, com $60 \mathrm{~cm}$ de coluna d'água, pintados internamente com tinta epoxi preta. Os tanques foram previamente lavados e desinfetados com solução de hipoclorito de cálcio $(2,5 \%)$, providos de circulação interna (filtro biológico), controle termostático $\left(28,0 \pm 1,1^{\circ} \mathrm{C}\right)$ e de fotoperíodo (12L:12E). A intensidade luminosa média incidente nos tanques foi de $6,3 \mu \mathrm{M} \cdot \mathrm{s}^{-1} \cdot \mathrm{m}^{-2}$ ( $\left.\pm 284 \mathrm{LUX}\right)$, considerada adequada para a reprodução de $M$. rosenbergii por CHAVES-JUSTO et al. (1990).

Devido a importância do sedimento na exteriorização e aderência dos ovos aos pleópodos nos crustáceos pleociematos (WiLlianson 1904), cada tanque recebeu uma camada de areia grossa, previamente lavada e fervida. O canibalismo durante a ecdise foi prevenido pela colocação de canos de PVC $(20 \mathrm{~cm} \times 4$ " $\varnothing)$ e substratos flutuantes de tela plástica, permitindo áreas de refúgio aos animais.

Os tanques foram providos com água de viveiros externos, utilizados na engorda de $M$. rosenbergii. A qualidade da água foi monitorada pela mensuração da temperatura (diária) e análise do $\mathrm{pH}$, amônio, nitrito e alcalinidade (semanal). A temperatura foi registrada por um termômetro com coluna de mercúrio e o pH com um peagômetro de precisão ( 0,01 unidades).

Os teores de amônio e nitrito foram analisados pelos métodos de Solorzano e Benschneider \& Robinson, respectivamente, ambos descritos em BAPTISTA et al. (1987). A leitura foi realizada com um espectrofotômetro e as concentrações expressas em $\mu \mathrm{g} / \mathrm{L}$, em função do íon nitrogênio $\left(\mathrm{NH}_{4}-\mathrm{N}_{\mathrm{e} \mathrm{NO}}-\mathrm{N}\right)$. A alcalinidade foi determinada pelo método descrito por GOLTERMAN et al. (1978), resultando valores em mg/L.

O método de ablação do pedúnculo ocular foi adaptado daquele descrito para camarões marinhos por PRIMAVERA (1985). As fêmeas foram retiradas da água e levemente enxugadas com papel toalha, tendo um ou ambos pedúnculos oculares extraídos com bisturi, rente à base. O local da incisão foi cauterizado "à quente" com um cauterizador elétrico, recebendo também marcação superficial em locais preestabelecidos no tergito de certos somitos abdominais para a identificação dos espécimes. Nos locais cauterizados foi ministrada uma mistura de pomadas antibióticas (Furacin ${ }^{\circledR}$ e Terramicina ${ }^{\circledR}$ ), na proporção 1:1. Todo o procedimento foi realizado em tempo hábil para minimizar o estresse dos animais. 
Durante as quatorze semanas que durou cada um dos experimentos, os animais de cada tratamento foram alimentados diariamente com lula e filé de peixe ( $5 \%$ da biomassa total) e observados diariamente no período vespertino (15:00 h), registrando-se a ocorrência de ecdise, presença de espermatóforo (cópula), desova, coloração da epiderme e comportamento alimentar. O estado de maturação gonadal de cada fêmea foi observado semanalmente, sendo classificado de acordo com RAO (1991) em três estágios: Imaturo (estágio I), em maturação (estágios II e III) e maturo (estágio IV). A dinâmica da maturação gonadal foi analisada para cada tratamento no decorrer dos dois experimentos realizados.

Durante o período experimental foi montado um ciclo de larvicultura com larvas provenientes de fêmeas submetidas ao processo cirúrgico, visando testar sua viabilidade e sobrevivência em sistema de circulação fechado.

Os valores médios de obtenção da primeira desova e período entre desovas foram comparados entre e dentro dos tratamentos de cada experimento por uma ANOVA. O mesmo procedimento foi empregado para os parâmetros da água em cada tratamento e o teste de Tukey utilizado para confirmar possíveis contrastes estatísticos entre as médias, sob o nível de significância de 1\% segundo SoKAL \& ROHLF (1979).

\section{RESULTADOS}

\section{Parâmetros abióticos da água}

Os parâmetros abióticos da água em cada tratamento estão representados pelas médias e respectivos desvios padrão na tabela $I\left(1^{\circ}\right.$ e $2^{\circ}$ experimentos). A temperatura e $\mathrm{pH}$ foram os parâmetros que mostraram menor variação durante $\mathrm{o}$ período estudado, contrastando com os teores de amônio e nitrito, que mostraram grande oscilação. Comparando-se as médias de alcalinidade da água para um mesmo tratamento, entre os dois experimentos realizados, percebe-se uma redução de aproximadamente $30 \%$ naqueles com camarões de sete meses de idade.

\section{Pigmentação epidérmica}

O padrão normal de coloração de $M$. rosenbergii, no ambiente proporcionado pelo presente experimento, pode ser verificado na figura $1 \mathrm{~A}$. Após a ablação os exemplares mostraram modificações no padrão cromático, principalmente nos urópodos e alguns segmentos dos quelípodos (mero, carpo e própodo). Os animais ablados unilateralmente apresentaram uma coloração mais escura que os exemplares do grupo controle, permanecendo com este padrão inalterado mesmo após a ecdise (Fig. 1B). Nos espécimes submetidos à ablação bilateral verificou-se a dispersão do pigmento vermelho um dia após o processo cirúrgico, conferindo-lhes coloração avermelhada. Este padrão permaneceu inalterado por algumas semanas, tornando-se mais alaranjado ao final do experimento (Fig. 1C).

\section{Atividade alimentar}

Foram observados três padrões distintos de comportamento alimentar. Os animais do grupo controle exploravam pouco a área do tanque, percebendo com facilidade a presença do alimento, que era rapidamente apreendido pelos quelípodos. A presença de restos de alimento no fundo do tanque foi freqüente. 

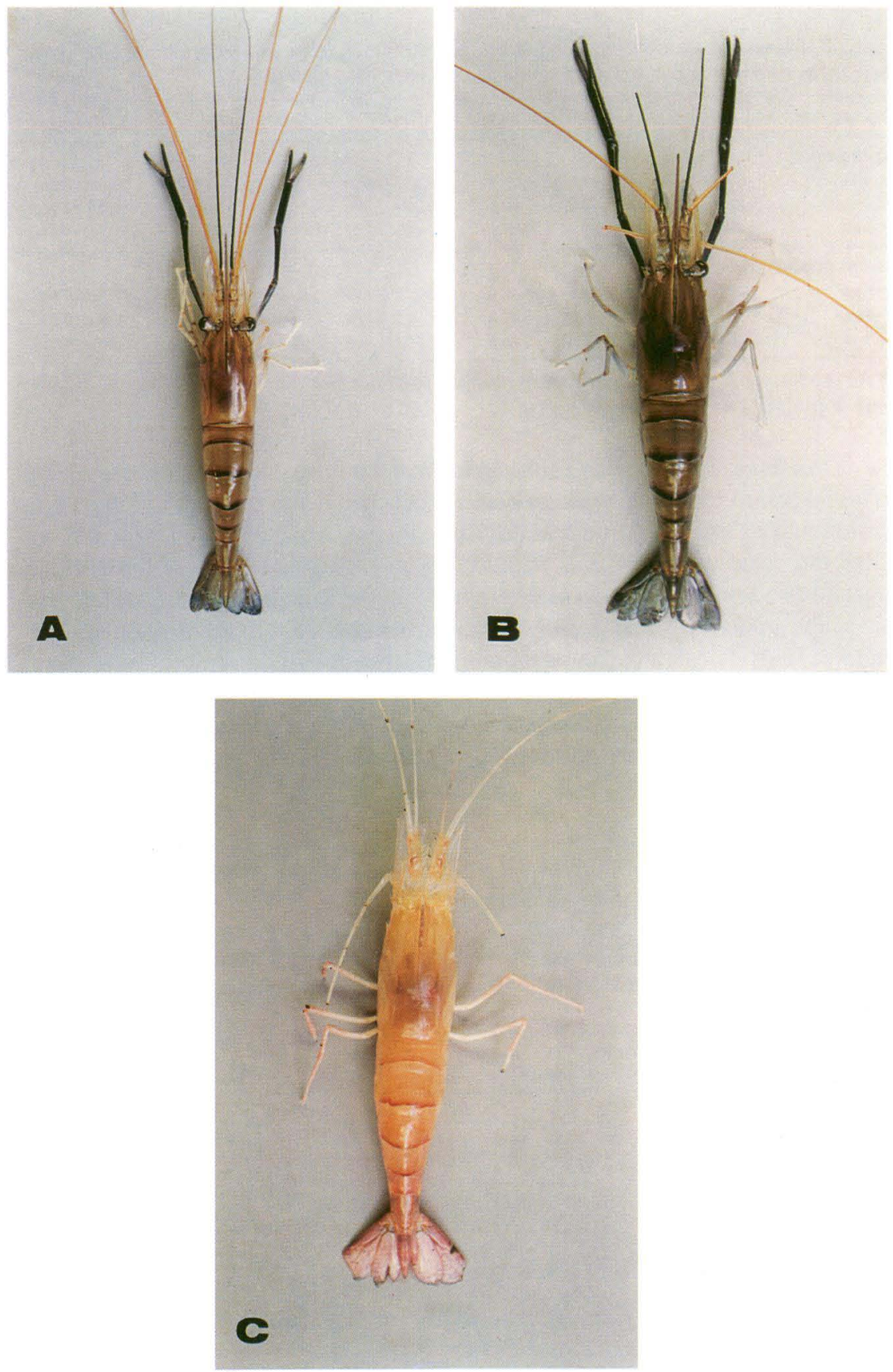

Fig. 1. Padrões de pigmentação epidérmica de exemplares de Macrobrachium rosenbergii, vista dorsal. (A) Grupo controle; (B) grupo uniablado; (C) grupo biablado. 
Tabela I. Parâmetros abióticos da água em cada tratamento (média \pm desvio padrão), registrados nos dois experimentos de ablação ocular de $M$. rosenbergii.

\begin{tabular}{|c|c|c|c|c|c|}
\hline Tratamentos & Temperatura $\left({ }^{\circ} \mathrm{C}\right)$ & Alcalinidade (mg/L) & $\mathrm{pH}$ & Amônia $(\mu \mathrm{g} / \mathrm{L})$ & Nitrito $(\mu \mathrm{g} / \mathrm{L})$ \\
\hline \multicolumn{6}{|l|}{ Experimento 1} \\
\hline Controle & $28,0 \pm 1,0 a$ & $67,20 \pm 3,40 a$ & $8,31 \pm 0,25 a$ & $9,5 \pm 4,3 a$ & $28,8 \pm 2,8 a$ \\
\hline Unilateral & $29,0 \pm 1,4 a$ & $70,20 \pm 5,32 a$ & $8,29 \pm 0,16 a$ & $15,0 \pm 11,0 a$ & $17,4 \pm 24,2 a$ \\
\hline Bilateral & $28,0 \pm 2,0 a$ & $78,38 \pm 3,42 a$ & $8,40 \pm 0,25 a$ & $10,0 \pm 4,3 a$ & $11,2 \pm 2,8 a$ \\
\hline \multicolumn{6}{|l|}{ Experimento 2} \\
\hline Controle & $28,5 \pm 0,7 \mathrm{a}$ & $42,24 \pm 10,48 a$ & $8,07 \pm 0,35 a$ & $14,0 \pm 19,4 \mathrm{a}$ & $12,7 \pm 20,5 a$ \\
\hline Unilateral & $29,0 \pm 1,4 a$ & $46,64 \pm 6,47 a$ & $8,50 \pm 0,43 a$ & $15,0 \pm 19,2 a$ & $9,5 \pm 14,9 \mathrm{a}$ \\
\hline Bilateral & $28,5 \pm 0,3 a$ & $57,64 \pm 9,96 a$ & $7,98 \pm 0,34 a$ & $34,0 \pm 21,2 b$ & $33,0 \pm 25,8 a$ \\
\hline
\end{tabular}

$\left(^{*}\right)$ As médias de um mesmo parâmetro abiótico seguidas por uma mesma letra não diferem entre si $(p>0,01)$.

As fêmeas submetidas à ablação unilateral utilizavam as tocas menos que as do grupo controle, ficando mais expostas sobre o sedimento arenoso. Sua percepção do alimento e a exploração da área do tanque durante a alimentação foram maximizadas em relação àquelas não submetidas ao processo cirúrgico. Dificilmente constatou-se a presença de restos de alimento no fundo do tanque neste tratamento.

Os animais biablados utilizaram pouco a área de refúgio disponível, mantendo-se imóveis sobre o sedimento arenoso a maior parte do tempo. No entanto, percebiam prontamente a presença do alimento, passando a buscá-lo por todo o tanque, muitas vezes chegando até a superfície. Todas as fêmeas deste tratamento apresentavam ingestão contínua de alimento, até que o limite físico de seus estômagos fosse atingido.

\section{Reprodução}

Os resultados reprodutivos e a sobrevivência no primeiro e segundo experimentos podem ser observados na tabela II.

Tabela II. Avaliação do desempenho reprodutivo e taxa de sobrevivência de fêmeas de $M$. rosenbergii com quatro meses de idade $\left(1^{\circ}\right.$ experimento) e sete meses de idade $\left(2^{\circ}\right.$ experimento).

\begin{tabular}{|c|c|c|c|c|c|c|c|c|c|c|c|c|c|}
\hline \multirow[t]{2}{*}{ Tratamentos } & \multirow{2}{*}{$\begin{array}{c}\mathrm{N}^{\circ} \text { máximo } \\
\text { de desovas } \\
\text { consecutivas } \\
\text { por fêmea }\end{array}$} & \multicolumn{5}{|c|}{$\begin{array}{c}\text { Obtençäo da } 1^{\text {a }} \text { desova } \\
\text { (dias) }\end{array}$} & \multicolumn{5}{|c|}{$\begin{array}{l}\text { Periodo entre desovas } \\
\text { (dias) }\end{array}$} & \multirow{2}{*}{$\begin{array}{c}\text { Taxa de } \\
\text { desova } \\
(\%)\end{array}$} & \multirow{2}{*}{$\begin{array}{c}\text { Taxa de } \\
\text { sobrevivência } \\
\text { das fêmeas } \\
(\%)\end{array}$} \\
\hline & & $\mathrm{N}$ & Min. Máx. & $\bar{x}$ & \pm & $\mathbf{s}$ & $N$ & Min. Máx. & $\bar{x}$ & \pm & s & & \\
\hline
\end{tabular}

\section{Experimento 1}

\begin{tabular}{llllllllllll} 
Controle & 1 & 2 & 31 & 34 & $32,5 \pm 2,1 \mathrm{~b}$ & - & - & - & - & 40 & 40 \\
Unilateral & 3 & 4 & 12 & 22 & $18,7 \pm 4,6 \mathrm{a}$ & 7 & 21 & 34 & $28,5 \pm 4,8 \mathrm{a}$ & 80 & 60 \\
Bilateral & 1 & 2 & 21 & 23 & $22,0 \pm 1,4 \mathrm{~b}$ & - & - & - & - & 40 & 0 \\
\hline
\end{tabular}

\section{Experimento 2}

\begin{tabular}{llllllllllrrr} 
Controle & 2 & 5 & 34 & 37 & $36,4 \pm 2,5 \mathrm{~b}$ & 5 & 36 & 38 & $37,0 \pm 0,7 \mathrm{~b}$ & 100 & 100 \\
Unilateral & 3 & 4 & 15 & 36 & $27,7 \pm 9,3 \mathrm{~b}$ & 4 & 22 & 32 & $27,5 \pm 2,6 \mathrm{a}$ & 80 & 60 \\
Bilateral & 2 & 1 & - & - & 17 & 1 & - & - & 21 & 20 & 0 \\
\hline
\end{tabular}

$\left({ }^{*}\right)$ As médias na coluna, seguidas pela mesma letra, não diferem estatisticamente entre si $(p>0,01)$. 
Quando comparada ao grupo controle a técnica de ablação unilateral proporcionou uma redução de $42,5 \%$ no tempo de obtenção da primeira desova para as fêmeas com quatro meses de idade e $23,9 \%$ para as fêmeas com sete meses. No primeiro caso, as médias mostraram contraste estatístico ( $\mathrm{p}<0,01)$, o que já não ocorreu no segundo experimento $(\mathrm{p}>0,01)$. Neste sentido, apesar da ablação bilateral ter promovido reduções expressivas em relação ao grupo controle, $32,3 \%\left(1^{\circ}\right.$ experimento) e $53,3 \%$ ( $2^{\circ}$ experimento), os valores médios obtidos para o primeiro experimento não foram estatisticamente distintos $(p>0,01)$.

Cerca de seis semanas após o processo cirúrgico, as fêmeas uniabladas do segundo experimento foram acometidas por uma contaminação fúngica na massa ovígera, necessitando de tratamento antibiótico. Apesar disso, este grupo foi caracterizado pelo maior número de desovas consecutivas, mostrando a eficácia desse processo cirúrgico frente aos demais tratamentos. No primeiro experimento este fato não pôde ser evidenciado pela inexistência de desovas consecutivas nos grupos controle e biablado.

$\mathrm{Na}$ figura $2 \mathrm{~A}$ pode-se verificar que as fêmeas com quatro meses de idade, submetidas à ablação unilateral, mostraram uma antecipação da primeira desova perante os demais tratamentos realizados, os quais, por sua vez, não apresentaram contraste estatístico entre si $(p>0,01)$. Por outro lado, as médias do intervalo entre desovas, para as fêmeas uniabladas de diferentes idades, não mostraram contraste estatístico $(p>0,01)$, apesar de serem menores que as obtidas para as fêmeas com sete meses do grupo controle ( $\mathrm{p}<0,01)$ (Fig. $2 \mathrm{~B}$ ).

As fềmeas submetidas à ablação unilateral mostraram quatro incidências de maturação durante o período estudado (Fig. 3), correspondendo ao dobro do registrado para o grupo controle do primeiro experimento, embora um terceiro ciclo de maturação gonadal possa ser visualizado para as fêmeas com sete meses $\left(2^{\circ}\right.$ experimento). Nos dois tratamentos de ablação bilateral verificou-se apenas um ciclo de maturação gonadal, devido a mortalidade de todos exemplares após 42 dias do processo cirúrgico ( $26 \pm 16$ dias).

As pós-larvas provenientes de fêmeas abladas unilateralmente, apresentaram uma sobrevivência de $70 \%$ ao final do ciclo de larvicultura, não tendo sido observadas deformações.

\section{DISCUSSÃO}

\section{Parâmetros abióticos da água}

Durante todo o experimento os parâmetros abióticos da água permaneceram dentro dos padrões normais previstos para o cultivo de $M$. rosenbergii. A temperatura e o pH estiveram na faixa considerada ideal por VALENTI (1985) e NEW (1995), que varia de 28 a $31^{\circ} \mathrm{C}$ e 7,0 a 8,5 , respectivamente. O mesmo foi constatado para os níveis de alcalinidade, amônio e nitrito, que não excederam os limites de tolerância da espécie, que são de $180 \mathrm{mg} / \mathrm{L}, 2.000 \mu \mathrm{g} / \mathrm{L}$ e $500 \mu \mathrm{g} / \mathrm{L}$, respectivamente (SiPAÚBA-TAVARES 1994). Dessa forma acredita-se que os parâmetros abióticos da água não interferiram nos resultados obtidos no presente estudo.

Como a origem de água nos dois experimentos foi a mesma, a redução expressiva da alcalinidade nos tratamentos do segundo experimento pode ser decorrente do maior uso das reservas de carbonato pelos animais de maior porte na pós-muda, necessárias ao enrijecimento do novo exosqueleto. 

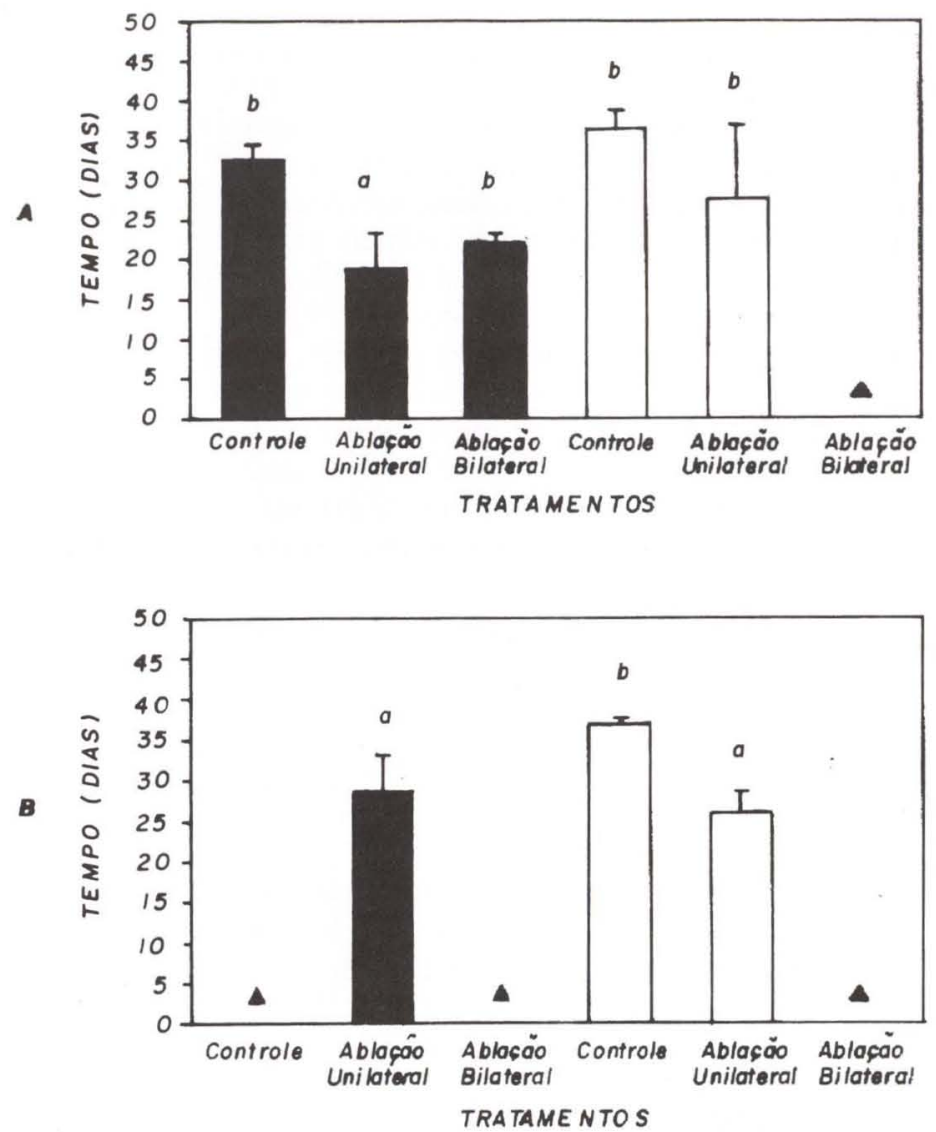

$\Delta$ = Ausáncia de dados ou repetiçóes
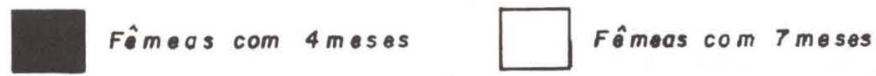

Fig. 2. Ocorrência da primeira desova (A) e intervalo entre desovas (B) das fêmeas de $M$. rosenbergii, submetidas a cada tratamento, nos dois experimentos realizados. Barra: média, linha: desvio padrão, letras associadas às barras: quando similares não mostram contraste estatístico ao nivel de significância de $1 \%$.

\section{Pigmentação epidérmica}

Os crustáceos decápodos apresentam adaptação de sua coloração ao ambiente em que se encontram (BROWN 1935; ROBISON \& CHARLTON 1973), tornando difícil a definição de um padrão específico. Apesar da capacidade de percepção de estímulos visuais pelos animais submetidos à ablação unilateral, verificou-se certa incapacidade de adaptação à cor da areia, apresentando uma tonalidade mais escura 
do que aquela do grupo controle. Tal fato era esperado em virtude da dispersão dos pigmentos vermelhos e amarelos, como mencionado para outros camarões por RUPPERT \& BARNES (1996), à exceção do que ocorre com Crangon spp. De acordo com BROWN et al. (1939), a ablação ocular na maioria das espécies de Palaemonidae promove uma dispersão generalizada de pigmentos nos cromatóforos, com prejuízo adaptativo à coloração do fundo, como constatado no presente estudo.

FÊEMAS COM 4 MESES

CONTROLE
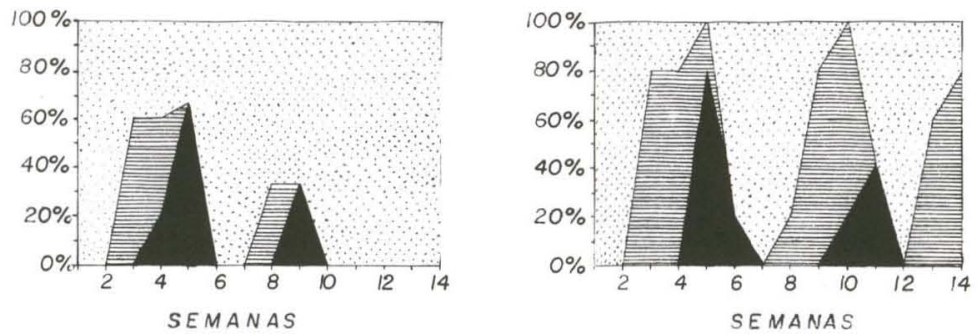

ABLACÃO UNILATERAL
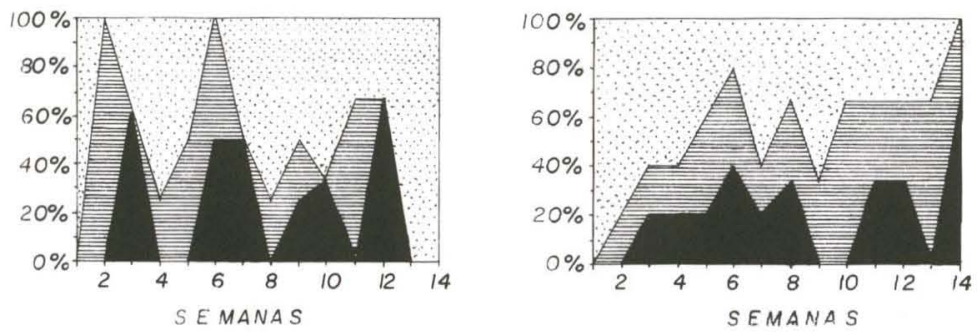

A B LACAOO BILATERAL
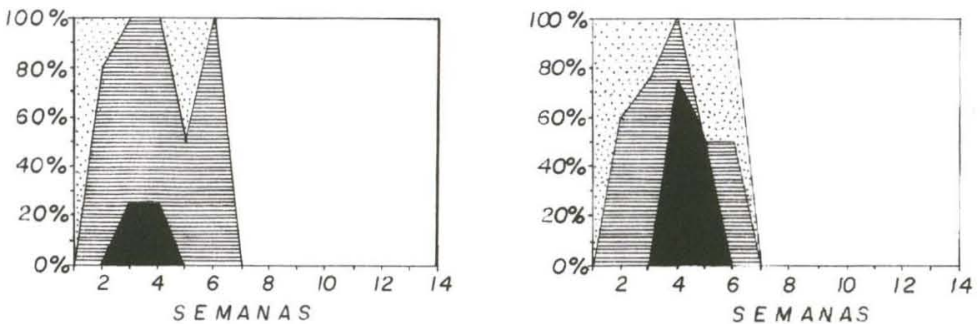

.7. IMATURAS EMMATURAGÃO MATURAS

Fig. 3. Gráficos de área representando a maturação gonadal das fêmeas de $M$. rosenbergii, em cada tratamento, durante os dois experimentos de ablação ocular. 
O padrão avermelhado, registrado para as fêmeas de $M$. rosenbergii submetidas à ablação bilateral, foi similar ao constatado por SCHEER \& SCHEER (1954) e KNOWLES et al. (1955) para o camarão Palaemon serratus (Pennant, 1977). Embora tais autores acreditassem que somente o hormônio concentrador do pigmento vermelho fosse produzido pelos pedúnculos oculares, outras espécies da Família Palaemonidae, e.g. Palaemonetes vulgaris (Say,1818) e Palaemon paucidens De Haan, 1844, apresentam-no, também, produzido nos tecidos extra-oculares, juntamente com o dispersor (BRown et al. 1952; FInGERMAN \& COUCH 1967; АоTO 1965). Tal fato evidencia que o hormônio concentrador do pigmento vermelho também ocorre nos tecidos extra-oculares nestas espécies, contrastando com $M$. rosenbergii, que mostra uma permanência da coloração vermelha nos exemplares biablados durante todo o experimento.

CASTILlo \& NEGRE-SARDARGUES (1991) verificaram que a remoção do pedúnculo ocular do ermitão Clibanarius erythropus Latreille, 1818, promove alterações no metabolismo do $\beta$-caroteno e astaxantina livre, responsáveis pela cor avermelhada resultante da oxidação do anidrido, que liga essas duas substâncias.

\section{Atividade alimentar}

Um aumento na quantidade de alimento ingerido foi verificado em espécimes ablados das lagostas Panulirus homarus (Linnaeus, 1758) e P. ornatus (Fabricius, 1798), estudados por VIJAYAKUMARAN \& RADHAKRISHNAN (1984) e JUINIOMEÑEZ \& RUINATA (1996), respectivamente. O mesmo foi registrado para os camarões marinhos Penaeus monodon Fabricius, 1798 por PrIMAVERA (1988) e $P$. notialis Pérez Farfante, 1967 por RosAs et al. (1993).

De acordo com esses autores, a ablação ocular promove aceleração metabólica, cujo déficit energético é compensado pelo aumento da quantidade de alimento ingerido. Além disso, existe a possibilidade de $M$. rosenbergii produzir em seu pedúnculo ocular um fator inibidor da alimentação (FIA), como já registrado para o crustáceo Uca pugilator (Bosc, 1802), responsável pelo aumento da sensibilidade quimioreceptiva aos alimentos quando submetidos à ablação bilateral (SEARS et al. 1991). Segundo resultados desses últimos autores, $97 \%$ dos caranguejos biablados exibiram maior sensibilidade a baixas concentrações de glicose $(0,0625 \mathrm{M})$, contrastando com os animais não ablados, $67 \%$ dos quais apresentaram comportamento alimentar somente em concentração 16 vezes maior. Desta forma, a ablação bilateral eliminaria os dois centros produtores do FIA, causando alterações no padrão de alimentação e acentuando a percepção destes animais à presença do alimento.

\section{Reprodução}

A técnica de ablação unilateral foi o tratamento mais eficiente para $M$. rosenbergii, apresentando vantagens como: antecipação da primeira desova; aumento do número de desovas consecutivas; redução no período entre desovas; e obtenção de alta taxa de desova/sobrevivência ao final do experimento.

De acordo com Primavera (1978), a ablação em $P$. monodon promove a maturação gonadal somente a partir de cinco meses de idade, demonstrando a precocidade de $M$. rosenbergii, cujos resultados foram satisfatórios em indivíduos 
com apenas quatro meses. O registro das fêmeas ovígeras de $M$. rosenbergii, nos tanques experimentais do Setor de Carcinicultura do CAUNESP, tem ocorrido com comprimento total superior a $95 \mathrm{~mm}$, confirmando a eficiência da técnica de ablação unilateral, haja visto que os animais de quatro meses apresentavam cerca de metade desse tamanho. De forma similar, SAGI et al. (1997) obtiveram uma antecipação da primeira desova em fêmeas de Cherax quadricarinatus Von Martens, 1868, com sete meses de idade.

Os resultados reprodutivos obtidos no presente estudo corroboram outros, realizados anteriormente, que mencionam a ablação unilateral como melhor forma de promover a maturação gonadal por desequilíbrio endócrino (PANOUSE 1943; NURJANA \& YANG 1976; LAWRENCE et al. 1980; LUMARE 1981; BROWDY \& SAmocha 1985; Primavera 1988; SAgi et al. 1997).

A ablação unilateral de $M$. rosenbergii permitiu a obtenção de larvas e pós-larvas viáveis, corroborando os dados obtidos por NAGABHUSHANAM \& KulKarni (1983), BROWDY \& SAMOCHA (1985) e SAGI et al. (1997), para Parapeneopsis hardwickii (Miers, 1878), Penaeus semisulcatus De Haan, 1844 e Cherax quadricarinatus, respectivamente.

No presente trabalho, apesar do grupo de ablação bilateral ter apresentado melhores resultados que $o$ controle, a mortalidade de todas as fêmeas, cerca de um mês após a cirurgia, indica a inviabilidade de seu uso. Fato similar já foi registrado para outros camarões marinhos biablados por SANTIAGo (1977) para Penaeus monodon e CHU \& CHOw (1992) para Penaeus chinensis (Osbeck, 1765). Tal inferência é corroborada por JUINIO-MEÑEZ \& RUINATA (1996) que verificaram uma menor taxa de sobrevivência para exemplares da lagosta Panulirus ornatus (Fabricius, 1798), submetidos à ablação bilateral. Por outro lado, GESTEIRA et al. (1989) estabeleceram a ablação bilateral como melhor resposta à maturação gonadal na lagosta Panulirus laevicauda (Latreille, 1817), possivelmente devido a maior resistência desta espécie ao processo cirúrgico.

Outro aspecto negativo, que inviabiliza a técnica de ablação bilateral para M. rosenbergii, foi a dificuldade de aderência dos ovos nas cerdas pleopodiais da fêmea, com interrupção do desenvolvimento embrionário. Caso semelhante foi descrito por CHOY (1987) para Penaeus canaliculatus (Oliver, 1811) submetidos à ablação unilateral, denotando a ocorrência de padrões interspecíficos resultantes da aplicação desta técnica.

\section{CONCLUSÕES}

A técnica de ablação unilateral do pedúnculo ocular mostrou-se mais eficiente na indução do processo de maturação gonadal, antecipação da desova e diminuição do intervalo entre desovas em fêmeas de Macrobrachium rosenbergii. Essa técnica pode ser aplicada mesmo em animais que ainda não atingiram a maturidade sexual, proporcionando bons resultados. A ablação bilateral provocou alta mortalidade e alterações expressivas nos padrões de alimentação e coloração, tornando-se inviável para a maturação desta espécie. 
AGRADECIMENTOS. Aos técnicos do Setor de Carcinicultura do CAUNESP, Biól. José Roberto Polachini e Sr. Valdecir Fernandes Lima, pelo precioso auxilio durante a fase experimental deste trabalho. À Profa. Dra. Maria Lucia Negreiros-Fransozo (Departamento de Zoologia, IB/UNESP, Botucatu) e Dr. Jelly Makoto Nakagaki (UEMS), pelas preciosas sugestões fornecidas por ocasião da defesa do trabalho de graduação que resultou no presente artigo.

\section{REFERÊNCIAS BIBLIOGRÁFICAS}

ADIYODI, K.G. \& R.G. ADIYODI. 1970. Endocrine control of reproduction in decapod crustacea. Bio. Rev. Cambriged Philos Soc. 45: 121-165.

Аото, T. 1965. On the possible participation of the nauplius-eye in chromatophoral regulation in the adult prawn, Palaemon paucidens. Jour. Fac. Sci. Hokkaido Univ., Ser. 6, (15): 652-661.

Baptista, J.M.R.; M.G.Z. Baumgarton \& L.F.H. Nilincheskil. 1987. Caderno de Análises em Oceanografia Química. Rio Grande, Editora FURG, Série Documento n 8, 64p.

BROWDY, C.L.\& T.M. SAMOCHA. 1985. The effect of eyestalk ablation on spawning, moulting and mating of Penaeus semisulcatus De Hann. Aquaculture 49: 19-29.

Brown JR., F.A. 1935. Color changes in Palaemonetes. Jour. Morphol. 57: 317-333.

Brown Jr., F.A.; H.E. Ederstron \& H.H. SCUd^More. 1939. Sinus, glandectomy in crustaceans sinus gland. Jour. Cell. Comp. Physiol. 15: 103-119.

Brown JR., F.A.; H.M. WeBB \& M.I. SANDEEN. 1952. The action of two hormones regulating the red chromatophores of Palaemonetes. Jour. Exp. Zool. 120: 391-420.

CAMPBELL, G.R. \& D.R. Fielder. 1986. Size at sexual maturity and occurrence of ovigerous females in three species of comercially exploited portunid crabs in S.E. Queensland. Proc. R. Soc. Queensl. 97: 79-87.

CASTILLO, R. \& G. Negre-SADARGUES. 1991. Effect of eyestalk ablation on the metabolism of carotenoid pigment of the hermit crab Clibanarius erythropus Latrielle (1818) (Crustacea, Decapoda, Anomura). Comp. Biochem. Physiol. 99B (1): 187-192.

Chaves-Justo, C.; K. Aid^ \& I. HAnyu. 1990. Effects of photoperiod and temperature on molting, reproduction and growth of the freshwater prawn Macrobrachium rosenbergii. Nippon Suisan Gakkaishi 52 (2): 209-217.

Choy, S. C. 1987. Growth and Reproduction of eyestalk ablated Penaeus canaliculatus (Oliver, 1811) (Crustacea: Penaeidae). Jour. Exp. Mar. Biol. Ecol. 112: 93-107.

CHU, K.H. \& W.K.CHOW. 1992. Effects of unilateral versus bilateral eyestalk ablation on moulting and growth of the shrimp, Penaeus chinensis (Osbeck, 1765) (Decapoda, Penaeidae). Crustaceana 62 (3): $225-233$.

DÉMEusY, N. 1962. Observations sur la maturation ovarienne du crabe Carcinus maenas L. aprés ablation des glandes de mue chez les femelles adultes. Premiers résultats. Acad. Sci. Paris: 3062-3064.

Drach, P. \& C. TChernigovtZeFf. 1967. Sur la méthode de détermination des stades d'intermue et son aplication générale aux crustacés. Vie et Milieu, Ser. A, 18: 595-610.

FingermAN, M. \& E.F. COUCH. 1967. The red pigment-dispersing hormone of the abdominal nerve cord and its contribution to the physiology of the prawn, Palaemonetes vulgaris. Rev. Can. Biol. 26: 109-117.

Gesteira, T.C.V.; J.R.F. Silva; E. Schrader \& A.V.P. Lima. 1989-90. Maturação de fêmeas da lagosta Panulirus laevicauda (Latreille) (Crustacea, Palinuridae) em confinamento. I. Influência da ablação do pedúnculo ocular. Arq. Ciên. Mar 28: 17-26.

Golterman, H.L.; R.S. Clymo \& M.A.M. OhnSTAD. 1978. Methods for physical and chemical analysis of freshwaters. London, Blackweel Sci., 214p.

JUINIO-MEÑEZ, M.A. \& J. RuinatA. 1996. Survival, growth and food conversion efficiency of Panulirus ornatus following eyestalk ablation. Aquaculture 146: 225-235.

Knowi.f., F.G.W.; D.B. Carlisl.F. \& M. Dupont-RaABe. 1955. Studies on pigment-activating 
substances in animals. 1. The separation by paper electrophoresis of chromactivating substances in arthropods. Jour. Mar. Biol. Assoc. U.K. 34: 611-635.

Kyomo, J. 1988. Analysis of the relationship between gonads and hepatopancreas in males and females of the crab Sesarma intermedia, with reference to resource use and reproduction. Mar. Biol. 97: 87-93.

Lawrence, A.L.; Y. Akamine; B.S. Middledictch; G. Chamberlain \& D. Hutchins. 1980. Maturation and reproduction of Penaeus setiferus in captivity. Proc. World Maricult. Soc. 1: 481-487.

LumAre, F. 1981. Artificial reproduction of Penaeus japonicus Bate as basis for the mass production of eggs and larvae. Jour. World Maricult. Soc. 12 (2): 335-344.

Meusy, J.J. \&. G.G. PAYEN. 1988. Female reproduction in Malacostracan Crustacea. Zool. Sci. 5: 217-265.

Nagabhushanam, R. \& G.K. Kulkarni. 1983. Reproductive biology of the female of a penaeid prawn Parapeneopsis hardwickii (Miers). Indian Jour. Fish. 29 (1, 2): 151-159.

NEw, M.B. 1990. Freshwater prawn culture: a review. Aquaculture 88: 99-143.

1995. Status of freshwater farming: a review. Aquaculture Research 26: 1-54.

NURJANA, M.L. \& W.T. YANG. 1976. Induced gonad maturation, spawning and postlarval production of Penaeus merguiensis De Man. Bull. Shrimp Cult. Res. Cent. 2 (3): 177-186.

PANOUSE, M.J. 1943. Influence de l'ablation du péduncule oculaire sur la croissance de l'ovarie chez la crevette Leander serratus. C.R. Acad. Sci. Paris 217: 533-555.

PASTEur, C. 1958. Influence de l'ablation de l'organe X sur le comportment chromatique de Leander serratus (Pennant). C.R. Hebd. Séances Acad. Sci. Paris 246: 320-322.

PAYEN, G.G. 1980-81. Aspects fondamentaux de l'endocrinologie de la reproduction chez les crustaces marins. Oceanis 6 (3): 309-339.

Primavera, J.H. 1978. Induced maturation and spawning in five-month-old Penaeus monodon Fabricius by eyestalk ablation. Aquaculture 13: 355-359.

. 1979. Notes on the courtship and mating behavior in Penaeus monodon Fabricius (Decapoda, Natantia). Crustaceana 37: 287-292.

PrIMAVERA, J.H. 1985. Review of maturation and reproduction in close thelycum penaeids. In: Y. TAKAI; J.H. Primavera \& J.A. Llobrera (Eds). International Conference on Culture of Penaeid Prawns and Shrimps. I. Proceedings Hoiolo, SAEFDEC Aquaculture Departament: 47-67.

- 1988. Maturation, reproduction, and broodstock tecnology. In: J.H. PRIMAVERA (Ed.). Biology and culture of Penaeus monodon. Tigbauan, Aquaculture Department of Southeast Asian Fisheries Development Center, 177p.

RAO, K.J. 1991. Reproductive biology of the giant freshwater prawn Macrobrachium rosenbergii (De Man) from Lake Kolleru (Andrha Pradesh). Indian Jour. Anim. Sci. 61 (7): 780-787.

RAO, K.R. 1985. Pigmentary Effectars, p. 395-462. In: D.E. Bliss; L.H. MANTEL (Ed.). The Biology of Crustacea. Integument, pigments, and hormonal processes. New York, Academic Press, Vol. 9, XXIX+550p.

Robison JR., W.G. \& J.S. CharLton. 1973. Microtubules, microfilaments and pigment granule movement in the cromatophores of Palaemonetes vulgaris (Crustacea). Jour. Exp. Zool. 186: 279-304.

Rosas, C.; I. Fernandes; R. Brito \& E. Diaz-Iglesia. 1993. The effect of eyestalk ablation on the pink shrimp, Penaeus notialis. Comp. Biochem. Physiol. 104A (1): 183-187.

RUPPERT, E.E. \& R.D. BARNES. 1996. Zoologia dos invertebrados. São Paulo, Roca, 1029p.

Sagi, A.; R. Shoukrun; T. Levy; A. Barki; G. Hulata \& I. Karplus. 1997. Reproduction and moult in previously spawned and first-time spawning red-claw crayfish Cherax quadricarinatus females following eyestalk ablation during the winter reproductive-arrest period. Aquaculture 156: 101-111.

Santiago JR., A.C. 1977. Successful spawning of cultured Penaeus monodon Fabricius after eyestalk ablation. Aquaculture 11: 185-196.

SCHFER, B.T. \& M.A.R. SCHF.ER. 1954. The hormonal control of metabolism in crustaceans. VII. 
Moulting and color change in the prawn, Leander serratus. Pubbl. Stn. Zool. Napoli 37: 397-418. SEARS, M.A.; D. RitTSChof \& T. O'Brien. 1991. Eyestalk factor modulation of chemically stimulated feeding in sand fiddler crabs Uca pugilator (Bosc, 1802). Jour. Exp. Mar. Biol. Ecol. 152: 1-13. SHiBLEY, G.A. 1968. Eyestalk function in cromatophore control in a crab, Cancer magister. Phisiol. Zool. 41: 268-279.

Sipaúba-Tavares, L.H. 1994. Limnologia Aplicada à Aqüicultura. Jaboticabal: FUNEP, 72p.

SOKAL, R.R. \& F.J. ROHLF. 1979. Biometria. Principios y métodos estadísticos en la investigación biológica. Madri, H. Blume Ediciones, 832p.

Steele, V.J.; B.H. Steele \& B.R. MAcPherson. 1977. The effect of photoperiod on the reproductive cycle of Gammarus setosus Dementieva, 1931. Crustaceana 4: 58-63.

TAN-FERmin, J.D. 1991. Effects of unilateral eyestalk ablation on ovarian histology and oocyte size frequency of wild and pond-reared Penaeus monodon (Fabricius) broodstock. Aquaculture 93: 77-86.

Valenti, W.C. 1985. Cultivo de Camarões de Água Doce. São Paulo, Ed. Nobel, 82p.

Vijayakumaran, M. \& E.V. Radhakrishnan. 1984. Effect of eyestalk ablation in the spiny lobster Panulirus homarus (Linnaeus): II. On food intake and conversion. Indian Jour. Fish 31 (1): 148-155.

Willianson, H.C. 1904. Contribuitions to the life histories of the edible crab (Cancer pagurus) and of other Decapod Crustacea: Impregnation: Spawning: Casting: Distribution: Rate of growth. $\mathbf{2 2}^{\text {nd }}$ Rep. Fish. Board Scotland, 3, Sci. Invest.: 100-140.

Recebido em 26.VIII.1999; aceito em 22.VIII.2000. 